\title{
Life under Supernovae. Story of the Cordillera Pine Forest
}

\author{
Alexey Ju. Retejum \\ Lomonosov Moscow State University, Leninskie Gory, Moscow, Russian Federation \\ Email: aretejum@yandex.ru
}

How to cite this paper: Retejum, A.J. (2019) Life under Supernovae. Story of the Cordillera Pine Forest. Open Journal of Geology, 9, 395-403.

https://doi.org/10.4236/ojg.2019.98026

Received: July 1, 2019

Accepted: August 11, 2019

Published: August 14, 2019

Copyright (C) 2019 by author(s) and Scientific Research Publishing Inc. This work is licensed under the Creative Commons Attribution International License (CC BY 4.0)

http://creativecommons.org/licenses/by/4.0/ (c) (i) Open Access

\begin{abstract}
The article presents the first direct evidence of the influence of supernova flashes on the biosphere. Geochemistry and paleontology have not yet provided convincing data on the life response to disasters in the Milky Way Galaxy. This gap was eliminated through tree ring analysis of bristlecone pine from the Cordilleras responded to seven supernova outbursts in 185-1604 $\mathrm{AD}$. The author used the superposed epoch method to process data at the 11 longest dendrochronologies, based on the results of measurement of annual growth of about 300 trees. The main finding is the growth depression in high-mountain population caused by supernova outbursts lasted for 20 - 30 years after the event. Moreover, in most cases, drastic growth reduction occurred one year prior to the event. In some cases, the annual tree ring increment exceeded the normal range many years after the event, and, consequently, it could be concluded that plant response was associated with the ozone layer depletion.
\end{abstract}

\section{Keywords}

Supernova, Bristlecone Pine Trees, Cordillera, Plant Response, Ozone Layer Depletion

\section{Introduction}

The important question of whether the biosphere, a product of solar activity, is affected or not affected by other Milky Way bodies, was positively answered as early as in 1950 by O. H. Schindewolf, a German paleontologist [1], who hypothesized about the impact of supernova explosions on the growth and development of living organisms. In recent years, when it was discovered that 60-iron of cosmic origin is widespread in the latest sediments on the ocean floor, the problem of dependence of life on supernovae attracts more and more attention 
[2]-[12]. However, so far only indirect evidence of their influence on evolution has been obtained, which is the coincidence of the time of isotope deposition with changes in environmental conditions and rearrangements within the flora and the fauna. The situation of uncertainty in reconstructions of the past is reflected in the expressions typical for the corresponding publications: "radiation from supernovae could have ionized the atmosphere", "ionization of the atmosphere by supernovae may lead", "supernovae could have altered", "explosions might have triggered mutations", "a supernova would have grievous effects", "supernovae may have played a little-known role", "an interesting coincidence" and so on. It is significant that the authors of the final report "Near-Earth Supernova Explosions: Evidence, Implications, and Opportunities" submitted to the 2020 Decadal Survey on Astronomy and Astrophysics U.S. National Academies of Sciences, Engineering, and Medicine, were limited to a simple statement in the chapter "Possible Biological Effects", that "A supernova at $10 \mathrm{pc}$ would surely be very dangerous for the biosphere, but a distinctive signature remains to be found in the geological record" [13].

The first step towards the identification of traces of a supernovae on Earth did B.P. Konstantinov and G.E. Kocharov [14]. In 1965 they proposed to reconstruct the modulation parameters of galactic cosmic rays from the ${ }^{14} \mathrm{C}$ and ${ }^{10} \mathrm{Be}$ records. This idea encouraged a pioneer study by N.V. Lovelius on the response of the Turkestan juniper ( $J$. turkestanica Kom.) to recent supernova outbursts [15]. Nevertheless, the studies in this direction were suspended for more than 30 years. In $1974,{ }^{14} \mathrm{C}$ content was determined in the annular rings of 400 -year-old trees, and these data demonstrated sharp increases corresponding in time to SN 1572 and SN 1604 supernovae [16], thus confirming the possibility of detecting the supernovae remnants in the planet envelopes. Shortly afterwards, due to determination of ${ }^{10} \mathrm{Be}$ content in the Greenland ice core [17], signs of supernova explosion that occurred 10,000 to 40,000 years ago were found, and this supernova was very close to the Solar system [ca. 150 light years] [18] [19]. Additional information on ${ }^{10} \mathrm{Be},{ }^{14} \mathrm{C}$, and ${ }^{36} \mathrm{Cl}$ levels definitely confirmed the hypothesis of a supernova outburst occurring about 35,000 years ago [20] [21]. Later it has been found that galactic cosmic rays bring Fe isotopes to the Earth [22] [23], and abnormally high concentrations of this atom may correspond to accelerated biota speciation [24] [25]. Currently available methods of fauna reconstruction, however, are not sufficiently accurate to give data for an unambiguous explanation of temporal coincidence of geochemical and paleontological changes. Obviously, direct evidence is required to confirm real existence of the phenomenon that is similar in nature to the effect predicted by O. H. Schindewolf. The results of the study of the modern forest [26] indicate the possibility of solving this problem.

It took a long time to go from properly designed experiments with very scarce data at hand to the discovery of most convincing evidence, that is, tree response as recorded in the pattern of annual rings. Below follows the first description of the consequences of the seven known events of cosmic ray generation during the period of 185-1604 based on the analysis of more than 1000 tree ring series from 
the longest-living plant species, i.e., bristlecone pine (Pinus longaeva D.K. Bailey). The analysis revealed previously unknown reactions of living organisms in the long period after the supernova outbreak and in the year before the outbreak, which indicates the existence of some new type of energy for science.

\section{Material and Methods}

As of now, a group of seven different supernova explosions, all going on at the same time, has been rather definitely identified by the criteria of long and multiple observations, fixed position in the firmament, unusual brightness, small galactic latitude, and the existence of remnants in the Milky Way (Table 1).

SN 1006 was the brightest observed supernova in recorded history, reaching in brightness a quarter or even half of the moon and giving a shadow in the daytime. SN 1054 was also seen during the daytime and exceeded roughly sixteen times the brightness of Venus.

The impact hypotheses testing consisted of the following stages: 1) The choice of an indicator that should have a long life expectancy and high sensitivity to changes in habitat, 2) Response detection, 3) Verification of the significance of the established ties, 4) The creation of the generalized model, 5) Explanation of the discovered patterns.

Pinus longaeva D.K. Bailey, a long-living species of bristlecone pine tree found in the Cordilleras, is the best object to examine for effects of supernova explosions in the biosphere. In this case, several dendrochronologies derived from mainly living ancient trees can be used (Table 2), thereby improving the conclusion validity. Arid climate and mountain habitat of the species increase the chance for the cosmic signals to be captured.

Generalized tree-ring data were analyzed by the method of Superposed Epoch Analysis [33]. Tree ring width values were averaged for each individual epoch and for all populations.

\section{Results}

As follows from the analysis of the generalized data, the supernova outbursts are

Table 1. Well known supernovae.

\begin{tabular}{|c|c|c|c|c|c|}
\hline Year & Constellation & Visibility period & $\begin{array}{l}\text { Apparent visual } \\
\text { magnitude (brightness) }\end{array}$ & Distance, ly & Place of observation \\
\hline 185 & Centaurus & $>20$ months & from -2 to -8 & $3000-8000$ & China, Korea \\
\hline 393 & Scorpios & 8 months & From 0 to -3 & $4000-34,000$ & China \\
\hline 1006 & Lupus & $>3$ years & from -7 to -9 & 7000 & $\begin{array}{c}\text { China, Japan, Egypt, Khwarezm, Iraq, } \\
\text { Morocco, Yemen, Switzerland }\end{array}$ \\
\hline 1054 & Taurus & 21 months & -6 & $6000-7000$ & China, Japan, Near East \\
\hline 1181 & Cassiopeia & 6 months & From 0 to -1 & $6500-8500$ & China, Japan \\
\hline 1572 & Cassiopeia & 18 months & -4 & $7500-10,000$ & China, Korea, Europe \\
\hline 1604 & Ophiuchus & 12 months & -3 & 20,000 & China, Korea, India, Europe \\
\hline
\end{tabular}

Source: Data from [27]-[32]. 
Table 2. Number of pine trees studied.

\begin{tabular}{|c|c|c|c|c|c|c|c|c|c|}
\hline \multirow{2}{*}{ Chronology } & \multirow{2}{*}{ Originator } & \multirow{2}{*}{ Place } & \multicolumn{7}{|c|}{ Supernova } \\
\hline & & & 185 & 393 & 1006 & 1054 & 1181 & 1572 & 1604 \\
\hline Methuselah Walk & D.A. Graybill & California & 27 & 26 & 29 & 31 & 33 & 55 & 54 \\
\hline Sheep Mountain & D.A. Graybill & California & 6 & 14 & 22 & 21 & 28 & 38 & 39 \\
\hline White Mountains & $\begin{array}{l}\text { C.W. Ferguson, E. } \\
\text { Schulman, H.C. Fritts }\end{array}$ & California & 9 & 13 & 15 & 16 & 15 & 13 & 31 \\
\hline Indian Garden & D.A. Graybill & Nevada & 21 & 19 & 22 & 18 & 20 & 26 & 27 \\
\hline Hill & D.A. Graybill & Nevada & 6 & 14 & 27 & 27 & 29 & 36 & 35 \\
\hline Mammoth Creek & D.A. Graybill & Utah & 3 & 4 & 19 & 20 & 29 & 31 & 32 \\
\hline Spring Mountains Lower & D.A. Graybill & Nevada & - & 2 & 19 & 22 & 26 & 36 & 36 \\
\hline \multirow[t]{2}{*}{ Wild Horse Ridge } & D.A. Graybill & Utah & - & 3 & 18 & 17 & 18 & 23 & 23 \\
\hline & Total: & & 72 & 95 & 171 & 172 & 198 & 258 & 277 \\
\hline
\end{tabular}

Source: National Centers for Environmental Information [https://www.ncdc.noaa.gov/data-access/paleoclimatology-data/datasets/tree-ring].

preceded by serious deterioration in the state of bristlecone pines, which is then followed by a one-year rise, and by a long-term depression thereafter (Figure 1(a)). Indices of radial growth, determined by more than 1200 tree rings, have significant differences during the outbreak time and previous years (Figure $1(b))$.

Drastic growth rate variations prior to and immediately after supernova appearance should be considered a characteristic tree response, since such changes are noted in $86 \%$ series. Naturally, this abnormality was maximally expressed (up to 73\%) during SN 1006, the most powerful outburst, and recorded in all the populations studied. Post-outburst annual growth was always characterized by significant negative trends (Figure 1(c)). These characteristics of plant response to the galactic signal are most prominent in the case of SN 1006 outburst (Figure 1(d)). Another supernova outburst, the second in power, had similar long-term consequences, that is, growth depression (Figure 1(e)). By comparing tree-ring chronologies, we could find out that the Methuselah Walk population of pine trees growing on dolomite-derived soils of the White Mountains, California, at an altitude of about $3000 \mathrm{~m}$ most completely reflects species sensitivity to the cosmic effect (Figure 1(f)). A similar effect of long-term tree growth depression can be seen from the chronologies of the awning pines, which are not so long, especially in the case of the SN 1006 outburst (Figure 1(g)).

\section{Conclusions}

In theory, three factors could affect the pine tree growth during post-supernova outbreak periods: variations in the solar activity, abnormal cosmic radiation, and atmosphere disturbance regimes. Values of ${ }^{10} \mathrm{Be}$ concentration [21] demonstrate that the level of total solar radiation did not change during supernova outbursts. The process of photosynthesis should have been depressed in response to 
high-energy ionizing particles and gamma quanta, as well as to UV-radiation, which is enhanced when the ozone layer is destructed. Coniferous trees are
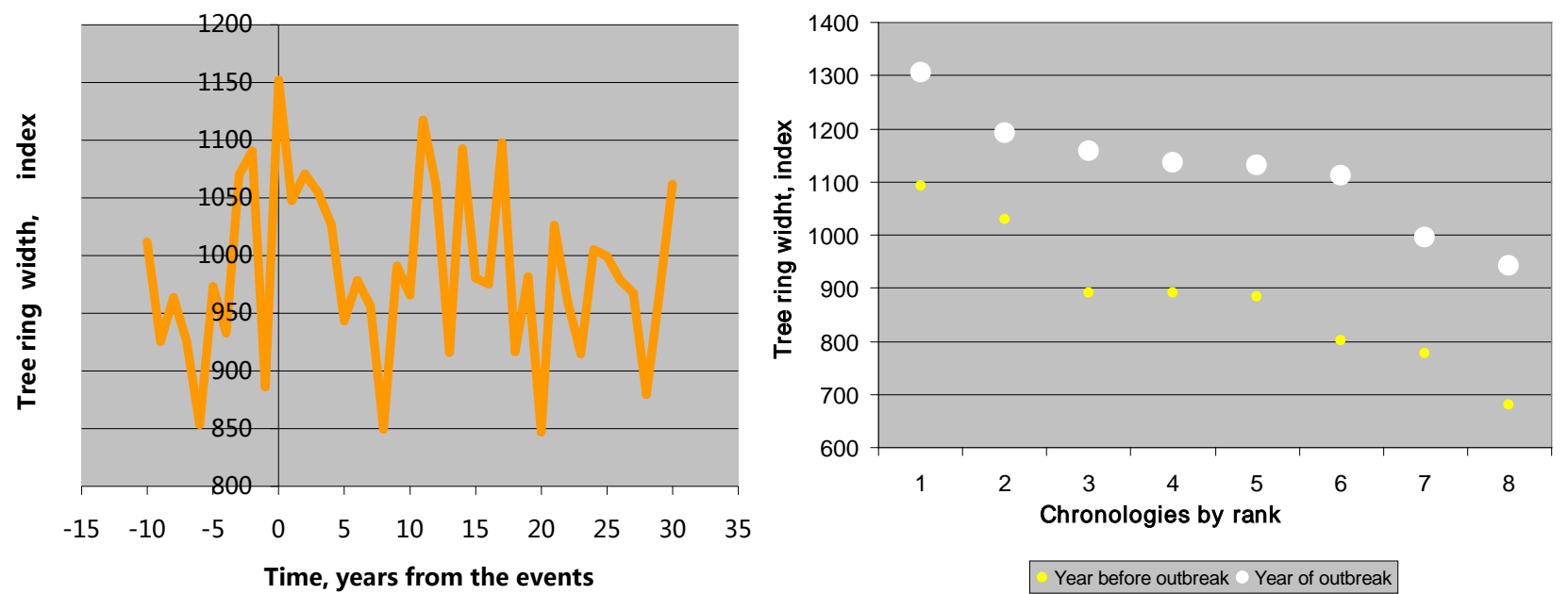

(a)

(b)

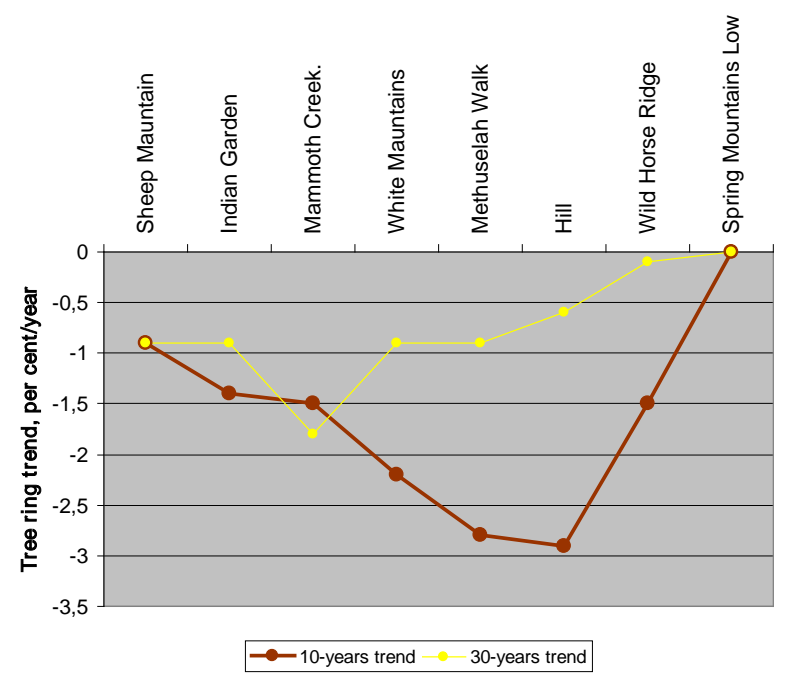

(c)

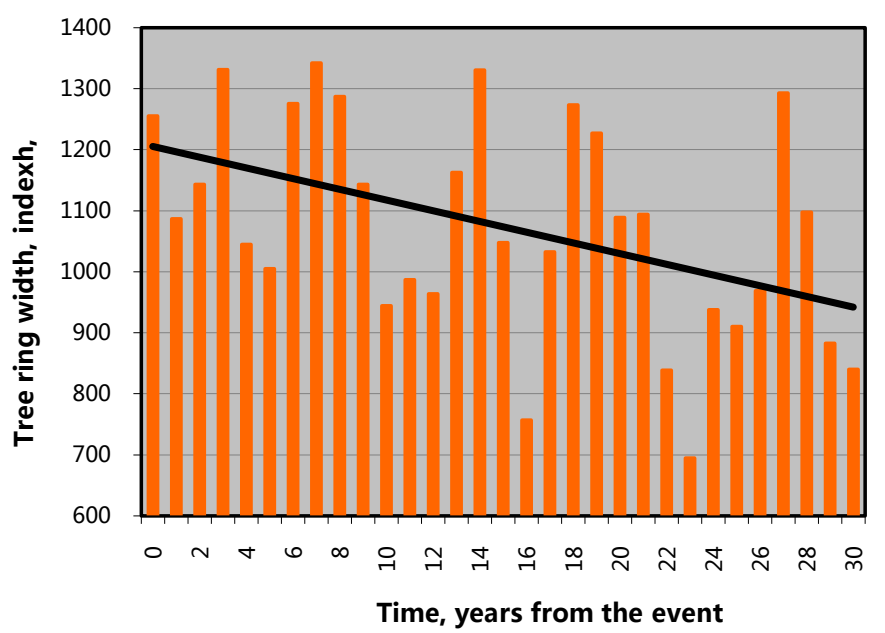

(e)

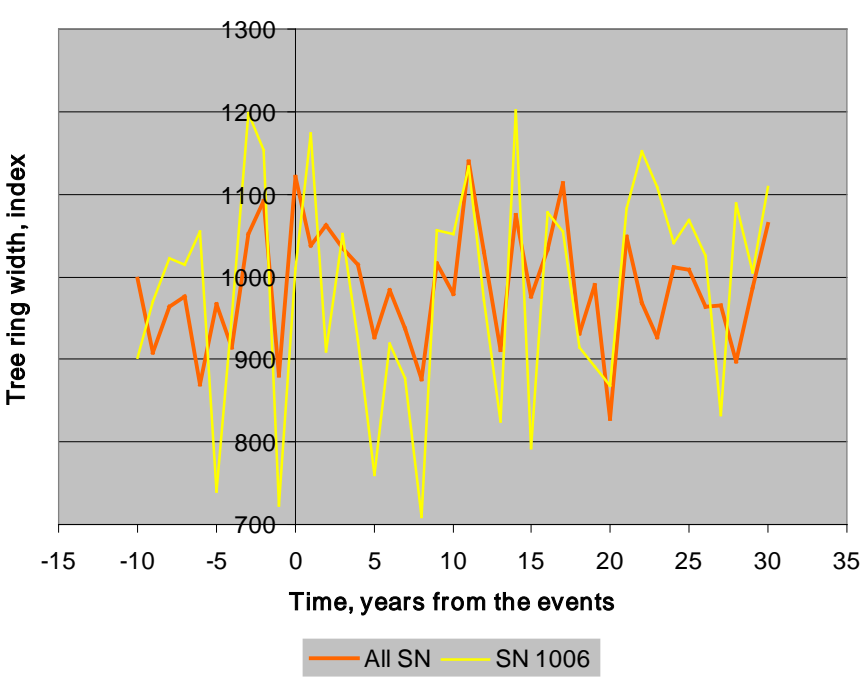

(d)

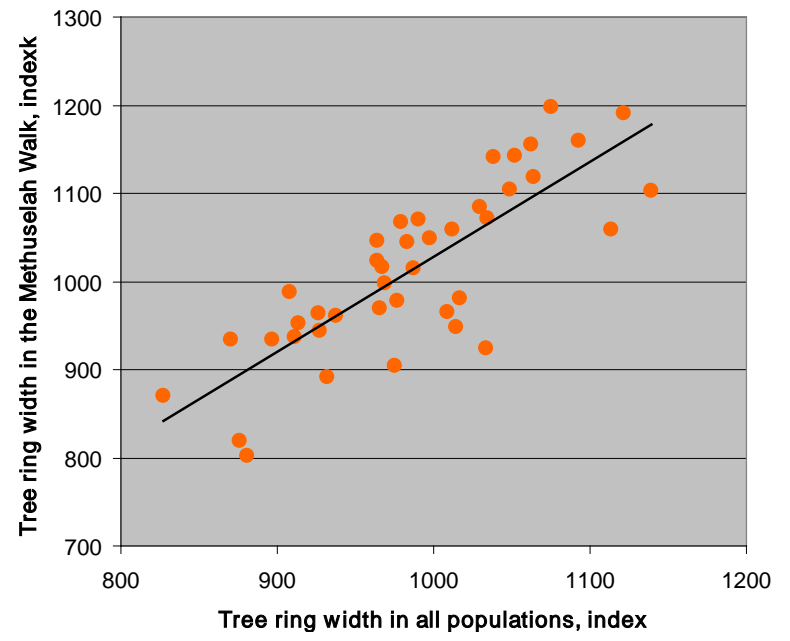

(f) 


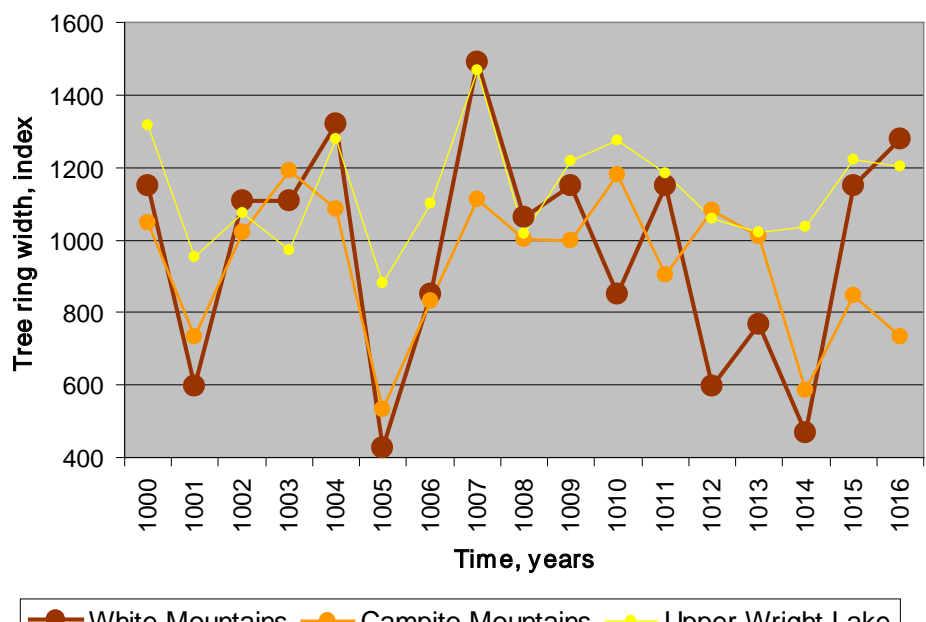

White Mountains - - Campito Mountains Upper Wright Lake

(g)

Figure 1. (a) General picture of annual tree growth before and after the supernova outburst compiled from all chronologies; (b) Tree ring width before and after the outbreaks. The difference is significant according the Mann-Whitney non-parametric U-test; (c) Tree growth trends in different populations; (d) Tree rings showing the effect of all SN and SN 1006. Synchronization is observed 1 year prior to the event; (e) Pine response to SN 185 outburst; (f) Correlation between annual increments in all populations and in the population of Methuselah Walk. Correlation coefficient 0.81; (g) Radial growth increment in the bristlecone pine tree of the White Mountains, the Campito Mountains and the Upper Wrigh Lake populations prior to and after SN 1006 outburst. Source: data-based calculations.

known to be devoid of resistance to such impacts. The most sensitive is needle rudiments, where the membrane systems of the cytoplasm and the nuclear chromatin-protein structures are damaged; mature needles age prematurely and die. As for pre-supernova outbreak, it is as yet unclear what causes regular decrease of tree growth. Apparently, we face a manifestation of the unknown energy. It should be added that the recently discovered dependence of the growth of modern forest on cosmic rays did not receive the necessary explanation [34].

The analysis of tree response to extreme cosmic impacts reveals certain years of excessive growth on the background of long-lasting growth depression, which may well be indicative of the predominance of reversible biological effects and the leading role of physical and chemical changes in the atmosphere. The results of examination of very old trees demonstrate pre- and post-actions of the supernova explosions, which are expressed in the short-term and long-lasting depression of their growth. These phenomena are associated with radiation of different nature. In the majority of dendrochronologies, the effects of SN 1006, the brightest of all supernovae, are most expressive.

Strong disturbances in the Earth's environment before and after the supernova explosions should have had the greatest impact on life in the biosphere.

\section{Acknowledgements}

The author is grateful to the International Tree-Ring Data Bank for the opportunity to use the archived data, to N.V. Lovelius for the initiative to conduct a 
dendrochronology study of the biosphere response to supernovae, to A. Shapiro for the information on the reconstructed values of the total solar irradiance and to D. Varlyguin for helpful comments.

\section{Conflicts of Interest}

The author declares no conflicts of interest regarding the publication of this paper.

\section{References}

[1] Schindewolf, O.H. (1950) Grundfragen der Paläontologie. Geologische Zeitmessung, Organische Stammesentwicklung, Biologische Systematic. Schweizerbart'sche Verlagsbuchhandlung, Stuttgart, 1-506.

[2] Thomas, B.C., Melott, A.L., Jackman, C.H., Laird, C.M., Medvedev, M.V., Stolarski, R.S., Gehrels, N., Cannizzo, J.K., Hogan, D.P. and Ejzak, L.M. (2005) Gamma-Ray Bursts and the Earth: Exploration of Atmospheric, Biological, Climatic, and Biogeochemical Effects. Astrophysical Journal, 634, 509-533. https://doi.org/10.1086/496914

[3] Thomas, B.C. and Honeyman, M.D. (2008) Amphibian Nitrate Stress as an Additional Terrestrial Threat from Astrophysical Ionizing Radiation Events? Astrobiology, 8, 731-733. https://doi.org/10.1089/ast.2007.0262

[4] Melott, A.L. and Thomas, B.C. (2009) Late Ordovician Geographic Patterns of Extinction Compared with Simulations of Astrophysical Ionizing Radiation Damage. Paleobiology, 35, 311-320. https://doi.org/10.1666/0094-8373-35.3.311

[5] Svensmark, H. (2012) Evidence of Nearby Supernovae Affecting Life on Earth. Monthly Notices of the Royal Astronomical Society, 423, 1234-1253. https://doi.org/10.1111/j.1365-2966.2012.20953.x

[6] Horvath, J.E. and Galante, D. (2012) Effects of High-Energy Astrophysical Events on Habitable Planets. International Journal of Astrobiology, 11, 279-286. https://doi.org/10.1017/S1473550412000304

[7] Thomas, B.C., Neale, P.J. and Snyder II, B.R. (2015) Solar Irradiance Changes \& Photobiological Effects at Earth's Surface Following Astrophysical Ionizing Radiation Events. Astrobiology, 15, 207-220. https://doi.org/10.1089/ast.2014.1224

[8] Neale, P.J. and Thomas, B.C. (2016) Solar Irradiance Changes and Phytoplankton Productivity in Earth's Ocean Following Astrophysical Ionizing Radiation Events. Astrobiology, 16, 245-258. https://doi.org/10.1089/ast.2015.1360

[9] Thomas, B.C., Engler, E.E., Kachelrieß, M., Melott, A.L., Overholt, A.C. and Semikoz, D.V. (2016) Terrestrial Effects of Nearby Supernovae in the Early Pleistocene. Astrophysical Journal Letters, 826, L3. https://doi.org/10.3847/2041-8205/826/1/L3

[10] Thomas, B.C., Goracke, B.D. and Dalton, S.M. (2016) Atmospheric Constituents and Surface-Level UVB: Implications for a Paleoaltimetry Proxy and Attempts to Reconstruct UV Exposure during Volcanic Episodes. Earth and Planetary Science Letters, 453, 141-151. https://doi.org/10.1016/j.epsl.2016.08.014

[11] Melott, A.L., Thomas, B.C., Kachelrieß, M., Semikoz, D.V. and Overholt, A.C. (2017) A Supernova at 50 Pc: Effects on the Earth's Atmosphere and Biota. Astrophysical Journal, 840, 105. https://doi.org/10.3847/1538-4357/aa6c57

[12] Thomas, B.C. (2017) Photobiological Effects at Earth's Surface Following a 50 pc Supernova. Astrobiology, 18, 481-490. https://doi.org/10.1089/ast.2017.1730 
[13] Astro2020 Science (2019) White Paper Near-Earth Supernova Explosions: Evidence, Implications, and Opportunities. Submitted to: The 2020 Decadal Survey on Astronomy and Astrophysics U.S. National Academies of Sciences, Engineering, and Medicine Committee on Astronomy and Astrophysics, $11 \mathrm{p}$.

[14] Konstantinov, B.P. and Kocharov, G.E. (1965) Astrophysical Phenomena and Radiocarbon. AN USSR Reports. (In Russian)

[15] Lovelius, N.V. (1974) On Possibility of Impact Assessment of Supernova Explosion on the Tree Growth. Botanical Journal, No. 7, 992-994. (In Russian)

[16] Kocharov, G.E., Dergachev, V.A., Sementsov, A.A., Romanova, E.N., Rumyantsev, S.A. and Malanova, N.S. (1974) Concentration of Radiocarbon in Tree Rings 1564-1583, 1593-1615, 1688-1712. Proceedings of the 5th Conference on Astrophysical Phenomena and Radiocarbon, Tbilisi, 4-6 October 1973, 47-60. (In Russian)

[17] Beer, J., Andrée, M., Oeschger, H., Stauffer, B., Balzer, R., Bonani, G., Stoller, C., Suter, M., Woelfli, W. and Finkel, R.C. (1983) Temporal ${ }^{10}$ Be Variations in Ice. Radiocarbon, 25, 269-278. https://doi.org/10.1017/S0033822200005579

[18] Kocharov, G.E. (1982) Burst of Cosmic Radiation and Cosmogenic Isotopes. In: Kocharov, G.E., Ed., Integrated Investigation of the Sun, Ioffe Physical-Technical Institute, Leningrad, 203-207. (In Russian)

[19] Konstantinov, A.N. and Kocharov, G.E. (1984) A 30,000 Year Record of the Cosmic-Ray Intensity. Pis' ma Astronomichesky Zhurnal, 10, 94-97. (In Russian)

[20] Sonett, C.P., Morfill, G.E. and Jokipii, J.R. (1987) Interstellar Shock Waves and 10/BE from Ice Cores. Nature, 330, 458. https://doi.org/10.1038/330458a0

[21] Konstantinov, A.N., Kocharov, G.E. and Levchenko, V.A. (1990) On the Supernova Explosion 35 ky Ago. Pis' ma Astronomichesky Zhurnal, 16, 799-803. (In Russian)

[22] Knie, K., Korschinek, G., Faestermann, T., Wallner, C., Scholten, J. and Hillebrandt, W. (1999) Indication for Supernova Produced ${ }^{60} \mathrm{Fe}$ Activity on Earth. Physical Review Letters, 83, 18. https://doi.org/10.1103/PhysRevLett.83.18

[23] Fimiani, L., Cook, D.L., Faestermann, T., Gómez-Guzmán, J.M., Hain, K., Herzog, G., Knie, K., Korschinek, G., Ludwig, P., Park, J., Reedy, R.C. and Rugel, G. (2016) Interstellar ${ }^{60} \mathrm{Fe}$ on the Surface of the Moon. Physical Review Letters, 116, Article ID: 151104. https://doi.org/10.1103/PhysRevLett.116.151104

[24] Breitschwerdt, D., Feige, J., Schulreich, M.M., Avillez, M.A., Dettbarn, C. and Fuchs, B. (2016) The Locations of Recent Supernovae near the Sun from Modelling ${ }^{60} \mathrm{Fe}$ Transport. Nature, 532, 73-76. https://doi.org/10.1038/nature17424

[25] Wallner, A., Feige, J., Kinoshita, N., Paul, M., Fifield, L.K., Golser, R., Honda, M., Linnemann, U., Matsuzaki, H., Merchel, S., Rugel, G., Tims, S.G., Steier, P., Yamagata, T. and Winkler, S.R. (2016) Recent Near-Earth Supernovae Probed by Global Deposition of Interstellar Radioactive ${ }^{60} \mathrm{Fe}$. Nature, 532, 69-72. https://doi.org/10.1038/nature17196

[26] Dengel, S., Aeby, D. and Grace, J. (2009) A Relationship between Galactic Cosmic Radiation and Tree Rings. New Phytologist, 184, 545-551. https://doi.org/10.1111/j.1469-8137.2009.03026.x

[27] Chu, S.-I. (1968) Supernovae from Ancient Korean Observational Records. Journal of the Korean Astronomical Society, 1, 29-36.

[28] Clark, D.H. and Stephenson, F.R. (1977) The Historical Supernovae. Pergamon Press, Oxford, New York.

[29] Stephenson, F.R. and Clark, D.H. (1976) Historical Supernovae. Scientific Ameri- 
can, 234, 100-107. https://doi.org/10.1038/scientificamerican0676-100

[30] Stephenson, F.R. and Green, D.A. (2003) A Millennium of Shattered Stars-Our Galaxy's Historical Supernovae. Sky and Telescope, 105, 40-48.

[31] Stephenson, F.R. and Green, D.A. (2005) A Reappraisal of Some Proposed Historical Supernovae. Journal for the History of Astronomy, 36, 217-229. https://doi.org/10.1177/002182860503600204

[32] Zhao, F.-Y., Strom, R.G. and Jiang, S.-Y. (2006) The Guest Star of AD185 Must Have Been a Supernova. Chinese Journal of Astronomy and Astrophysics, 6, 635-640. https://doi.org/10.1088/1009-9271/6/5/17

[33] Chree, C. (1913) Some Phenomena of Sunspots and of Terrestrial Magnetism at Kew Observatory. Philosophical Transactions of the Royal Society of London. Series A, Containing Papers of a Mathematical or Physical Character, 212, 75-116. https://doi.org/10.1098/rsta.1913.0003

[34] Shapiro, A.I., Schmutz, W., Rozanov, E., Schoell, M., Haberreiter, M., Shapiro, A.V. and Nyeki, S. (2011) A New Approach to Long-Term Reconstruction of the Solar Irradiance Leads to Large Historical Solar Forcing. Astronomy \& Astrophysics, 529, A67. 